\title{
Téoros
}

Revue de recherche en tourisme

\section{Géologie et tourisme : la collection d'items géologiques en tant qu'activité touristique}

\section{Daniel J. Boivin}

Volume 9, numéro 1, mars 1990

URI : https://id.erudit.org/iderudit/1080291ar

DOI : https://doi.org/10.7202/1080291ar

Aller au sommaire du numéro

Éditeur(s)

Université du Québec à Montréal

ISSN

0712-8657 (imprimé)

1923-2705 (numérique)

Découvrir la revue

Citer cet article

Boivin, D. J. (1990). Géologie et tourisme : la collection d'items géologiques en tant qu'activité touristique. Téoros, 9(1), 31-35.

https://doi.org/10.7202/1080291ar d'utilisation que vous pouvez consulter en ligne. 


\section{Remerciements}

J'aimerais remercier ici certaines personnes qui m'ont permis de mener à bien la préparation et la rédaction de cet article. Il s'agit de MM. Michel Gascon, André Brisebois et Tony Gordian (Club de Minéralogie de Montréal), Pierre Laroche et $\mathbf{M}$. Marois (Sociéte Minéralogique de la région de l'amiante), de M. Han Spoel (Montreal Gem \& Mineral Club).

\section{Introduction}

Le tourisme géologique peut être défini comme une activité spécialisée qui consiste à s'intéresser aux aspects géologiques des paysages et régions visitées. Il englobe beaucoup plus que la simple collecte d'échantillons. Par exemple, on peut considérer les activités suivantes comme faisant partie, de près ou de loin, de cette catégorie:

visite de parcs nationaux (Forillon); visite de musées géologiques et paléontologiques;

- visite d'attractions géologiques particulières comme:

- les chutes Montmorency ou Niagara; - les cavernes et grottes (caverne de la fée, St-Léonard):

. les cratères volcaniques (Hawaii); - les cratères de météorites (Meteor crater, Arizona);

- les visites de zones de geysers et de sources chaudes (Yellowstone,

Wyoming);

visite de mines et de carrières.

Le tourisme géologique s'appuie donc principalement sur une ou plusieurs ressources locales qui constituent une attraction pour une clientèle cible particulière. Quoique le potentiel touristique d'une région en cette matière $n^{*}$ est pas facilement quantifiable en termes de stratégies de marketing comme il est possible d'envisager dans d'autres

Monsieur Daniel J_ Boivin est géographe-aménagiste et accupe un poste de chercheur au Centre de Recherches en Amenagement ot en Developpo ment (CRADI à I'Université Laval. formes d'activités touristiques régionales, le tourisme géologique s'appuie d'abord sur une connaissance et une cartographie à la fois des formations géologiques régionales mais aussi des ressources naturelles exploitées ou exploitables. C'est ainsi qu'une attraction géologique est d'abord identifiée dans un rapport géologique; cette attraction peut être d'un intérêt extrềnement limité ou encore d'intérêt général. Dans le premier cas, il peut s'agir de formes ou de phénomènes géologiques particulièrement bien visibles mais d'un intérêt plus pédagogique que touristique. C'est ainsi que certaines régions ousites sont fréquemment visites par des groupes scolaires universitaires (Chutes Montmorency, rivages du fleuve à Neuville ou à 1'Islet-surMer, Cap-Diamant, Montérégiennes, etc.). Quant à ladeuxième catégorie d'attractions, comme ceux décrits plus haut, ils sont d'intérết gếnéralet susceptibles d'intéresser une plus vaste gamme d'individus.

Le présent article désire dresser un premier portrait des activités de tourisme au Québec reliées à la géologie. De façon plus spécifique, nous étudierons la collection d'items géologiques tels que minéraux et roches ainsi que les endroits au Québec qui sont particulièrement intéressants pour ces amateurs et professionnels. Un autre article est en préparation sur les fossiles. C'est dans le cadre d"une activité de loisir scientifique que nous aborderons les liens avec le tourisme.

\section{Portrait des activités}

La collection d'objets géologiques constitue une activité reliée au loisir scientifique. Il est utile de mentionner que ce loisir se caractérise par deux périodes d'activités dans l'année en rapport avec la présence ou l'absence de neige au sol. Puisque la collection nécessite une recherche au niveau du sol, la présence de neige constitue un facteur plus limitatif que les temperatures froides. On constate en consequence une véritable différenciation des tâches dans 1'année. Ainsi, la période de l'année sans neige, soit de mai à la fin octobre est consacrée aux visites sur le terrain et à la cueillette des échantillons alors que la période d'hiver est réservée au travail à la maison ou en laboratoire. $\mathrm{C}^{\prime}$ est donc surtout les saisons du printemps, de l'été et de l'automne qui retiendrons surtout notre attention en ce qui concerne les déplacements.

Ce loisir scientifique consiste essentiellement à se rendre sur des sites où l'on peut trouver des pièces géologiques dans le but de les collectionner ou de les mettre en valeur. Ainsi, certains amateurs se contenteront de cueillir les échantillons qu'ils trouvent, de les nettoyer, de les identifier, de les classifier et de les exposer d'une façon ou d'une autre; ce sont les minéralogistes ou pétrographes amateurs ou encore, dans le cas plus précis des fossiles, des paléontologues amateurs. Certains autres vont pousser leur travail dans l' art lapidaire et la gemmologie ou encore la sculpture et vont travailler la pierre pour la mettre en valeur (lire Brillant, J. et al., 1986). Ainsi, la création de bijoux artisanaux à partir d'échantillons bruts collectés eux-mêmes constitue une activité de loisir qui se développe de plus en plus. A partir d'intérêts personnels très différents, la phase de cueillette demeure néanmoins une activité pratiquée par la plupart et ceci de manière très semblable. Il va de soi que les tailleurs professionnels ou avancés choisiront $\mathrm{d}^{4}$ acheter leur matériel brut sur lequel ils travaillent plutôt que d'aller le chercher eux-mêmes. Il n'en demeure pas moins que tous sont tentés un jour ou l'autre d'aller voir par eux-mêmes des sites de collecte intéressants. Ceci est d'ailleurs valable autant en art lapidaire qu'en sculpture par exemple.

Les objets géologiques recherchés par les collectionneurs sont très diversifiés et peuvent adopter des formes variées. Une description sommaire $s$ 'impose afin de donner une idée au lecteur de quel matériel il s 'agit.

En matière d'abord de minéraux, le collectionneur recherche deux types d'echantillons soit les pièces cristallisées et les pièces massives. Les premiers sont très recherchés pour leur beauté et leur relative rareté. 
Des cristaux de quartz translucides, uniques ou agglomérés ensemble, constitue un exemple d'une pièce recherchée comme on peut en trouver à St-Rémi d'Amherst dans les Laurentides ou encore à la carrière Adam à Lawrenceville. Parmi les cristaux les plus fréquemment trouvés au Québec figurent la pyrite, la molybdénite, la calcite, le quartz, le mica, les grenats almandin et grossulaire, l'analcime, la wéloganite, 1'aegyrine, la tourmaline et plusieursautres. Les minéraux en masses c'est-à-dire non cristallisés sont aussi recherchés comme matériau lapidaire. On recherche beaucoup la sodalite (bleue), la wilsonite (mauve), la péristérite (bleue pâle) et $1^{4}$ amazonite (verte) pour $n^{\dagger}$ en citer que quelques-unes.

En matière de roches, les pièces recherchées sont surtout destinées à la sculpture. Peu de gens s'intéressent à la collection d'objets pétrographiques en tant que telles sauf si celles-ci présentent un intérêt décoratif ou esthétique particulier. Ainsi, certaines stéatites (pierre à savon), serpentinites, granites graphiques et anorthosites sont appréciées autant en pétrographie qu'en sculpture. Les pierres les plus favorables en sculpture sont d'abord les pierres tendres puisqu'elles sont plus facilement travaillables. Les marbres peuvent aussi présenter un intérết non négligeable pour la fabrication artisanale de meubles design mais cette activité est fort peu répandue à cause du poids important des pièces impliquées.

\section{Sites visités au Québec}

L'un des premiers aspects à considerer est la localisation des destinations de ces collectionneurs. A cause de la diversité mais aussi à cause de la spécificité des objets recherchés en termes de beauté, de rareté et de disponibilite, on conçoit facilement que certains endroits précis sont visités alors qued'autres n'offrent qu'un intérêt général. La localisation deces sites different d'abord selon que l'on recherche des minéraux ou des roches. La raison en est que chacun de ces groupes d'objets géologiques ne se rencontrent pas nécessairement aux mêmes endroits et dans les mêmes formations géologiques. Ainsi, les minéraux se trouvent dans des carrières ou des mines (actives ou abandonnées) et à quelques occasions dans des affleurements. Les pierres, quant à elles, peuvent se trouver à tous ces endroits mais plus frequemment dans des carrières actives ou abandonnées.

La localisation et la description des sites de collecte peut être trouvée d'abord dans plusieurs documents publics publiés par le gouvernement fédéral dont les livres de Ann P. Sabina qui forment une collection d'ouvrages intitulés Roches et minéraux pour le collectionneur. Les rapports géologiques et rapports spéciaux publiés par le gouvemement du Québec (ministère des Richesses Naturelles a 1"époque et le ministère Énergie et Ressources maintenant) sont aussi une source importante d'informations de ce type. On trouve également des ouvrages très précis publiés par des organismes parapublics comme le Conseil de Développement du LoisirScientifique (voir Boivin, D.J. et M. Di Vergilio, 1987 et Boivin, D.J., 1985) ou encore par différents Clubs de géologues amateurs (Boisclair, J. et D.J. Boivin, 1985).

Il $n$ "est pas dans notre intention de lister tous les sites visités annuellement par ces amateurs. Nous proposons plutôt de s'attarder aux sites les plus visités et où des excursions sont régulièrement organisées. De cette façon, notre approche nous permettra d'estimer si ces destinations vedettes constituent de véritables centres d'attraction touristiques particuliers et ainsi d'en chiffrer l'importance. Afin de localiser ces endroits, les tableaux 2 et 3 donnent les lieux visités annuellement par deux des trois Clubs au Québec. Les données dont nous disposons remontent aux dernières années seulement. Une carte des destinations relevées dans les trois premiers tableaux a aussi été dressée (voir figure 1).

Il est utile de mentionner également que les destinations choisies par les géologues amateurs ne sont restreintes à l'intérieur de régions définies par des frontières politiques mais plutôt par la distance à parcourir (et ainsi par le temps requis) pour se rendre à des endroits intéressants.

En général, la majorité des excursions organisées officiellement par les Clubs sont des activités journalières avec départ le matin tôt et retour le soir. Afin de permettre un maximum d'heures de travail sur le site même, quatre heures de déplacement au total (aller-retour') constitue une limite journalière maximum. Au-delà de cechiffre, une nuitée est en général requise et l'excursion se prolonge sur deux ou plusieurs journées. L'analyse des dates des visites des excursions indique d'abord que la collecte d'Echantillons est principalement une activité de fin de semaine et ceci autant au printemps, à l'été ou à l'automne.

Dans la plupart des cas, on observe que l'activité de collecte d'échantillons géologiques est centrée autour de l'automobile personnelle et occasionnellement par l'usage d'autobus nolisés. La raison principale de ceci repose dans la flexibilité dont peuvent profiter les excursionnistes dans le choix de l'heure de retour. Mais aussi, le collectionneur a besoin d'espace de rangement autant pour ses outils au départ que pour les pièces qu'il rapporte. Le poids important des collectes additionné au poids des outils justifie même l'usage d'un véhicule de type wagonnette ou véhicule toutterrain robuste. L'autobus n'est alors requis que dans les cas peu fréquents de sites três éloignés ou lors de visites de musées. De plus, lors des excursions organisées par les clubs, une certaine forme de covoiturage (avec partage des frais) est pratiquée pour offrir la possibilite à tous de pratiquer leur activité préférée.

La pratique de ce loisir nécessite que les visiteurs prennent au préalable des arrangements avec les propriétaires des sites. Tout d'abord, les clubs prennent soin de contacter annuellement les propriétaires des terrains fréquemment visités afin d'obtenir leur permission de se rendre sur le terrain dans le but de ramasser des échantillons. Dans d'autres cas, les visiteurs non organisés demandent sur place la permission au propriétaire. Sur la plupart des sites, le propriétaire du terrain tolère ou autorise en permanence la venue de collectionneurs de ce genre. Certains autres exigent un prix d'entrée comme c'est le cas à la mine Orford Nickel Mine près de St-Denis-deBrompton, à la carrière Poudrette au Mont St-Hilaire ou à la mine Yates près d'Otter Lake (Outaouais). Les montants sont en général très bas et varient de quelques dollars à 10 s pour le mont St-Hilaire. De plus, citons le cas de la carrière Adam où, il y a quelques années de cela, le propriétaire demandait $3 \$$ de 1 "heure pour la cueillette dans sa carrière.

\section{Un loisir scientifique à développer}

À notre connaissance, aucune êtude spécifique n'a jamais été entreprise sur ce loisir scientifique en tant qu'activité touristique au Québec. ¿ l'heure actuelle, le club SOMIEA de Thetford Mines, en association avec le musée du collège de l'amiante, prépare un concept régional de tourisme géologique. Il serait bien intéressant de nous faire connaître dans un prochain numéro de Téoros les détails de leur projet.

En ce qui concerne, force nous est de constater que de plus en plus de gens s'intéressent aux richesses géologiques de nos régions. C'est d'ailleurs une des facettes que prend la redécouverte de la nature en tant que source d'épanouissement et de 
détente personnelle. Une des preuves concrètes de ceci réside dans l'augmentation significative du nombre de membres des clubs au Québec comme le démontre le tableau 2 pour le Montreal Gem \& Mineral Club. Ces données ne nous sont malheureusement pas disponibles pour les autres clubs qui manquent de ressources et de bénévoles actifs pour tenir ces renseignements à jour. En 1989, on compte plus de 775 membres pour les trois clubs étudiés. Il est bien important aussi de souligner que les activités de ces clubs, incluant les excursions, sont documentées d'un bon support scientifique avec conférences, sessions d'identification, cours divers, visites de musées, etc.. Certaines activités para-scientifiques comme l'art lapidaire constitue un lien intéressant entre l'art et la science.

Récemment, quelques membres ont soulevé un aspect conflictuel qui est relié à leurs activités sur le terrain. D'un côté, les pressions populaires pour l'amélioration et la protection de notre environnement obligent les compagnies minières à restaurer leursite d'extractionet à remettre enétat les terrains perturbés. De $1^{t}$ autre côté, les amateurs de roches et minéraux voient avec tristesse la disparition de leurs sites de collecte. Cette problématique est bien exprimée d'ailleurs par E. Robinson dans un article paru dans Mine \& Quarry Environment (vol. 3, no 1, 1989, pp. 28-30). Ainsi, la fermeture et la restauration d'un site aussi exceptionnel que la carrière Poudrette au Mont St-Hilaire serait perçue comme dramatique pour ces collectionneurs qui $s^{*} y$ rendent plus d'une fois par année.

En conclusion, nous espérons avoir apporté quelques éléments de réflexion sur une facette peu connue du tourisme régional en montrant que la géologie peut aussi être envisagée comme une activité touristique et que celle-ci est bel et bien pratiquée au Québec. $f$

\footnotetext{
RÉFÉRENCES

BOIVIN, D-J et M. DIVERGILIO, Guide du collectionneur = minéraux, fossiles et roches, Conseil de Developpement du Loisir Scientifique. Montreal. $1987,132 \mathrm{p}$.

BOISCLAIF, J. et D.J. BOVVIN, Minéraux du Mont StHilaire, Club de Minéralogie de Montreal, Montréal, octobre 1985, $40 \mathrm{p}$.

BOIVIN, D--J., Roches et minéraux du Québec: guide d'excursion pour le collectionneur, Conseil de Developpement du Loisir Scientifique, Montreal, juillet $1985,142 \mathrm{p}$.
}

Figure 1

Principaux sites d'excursions minéralogiques visités entre 1986 et 1989 au Québec

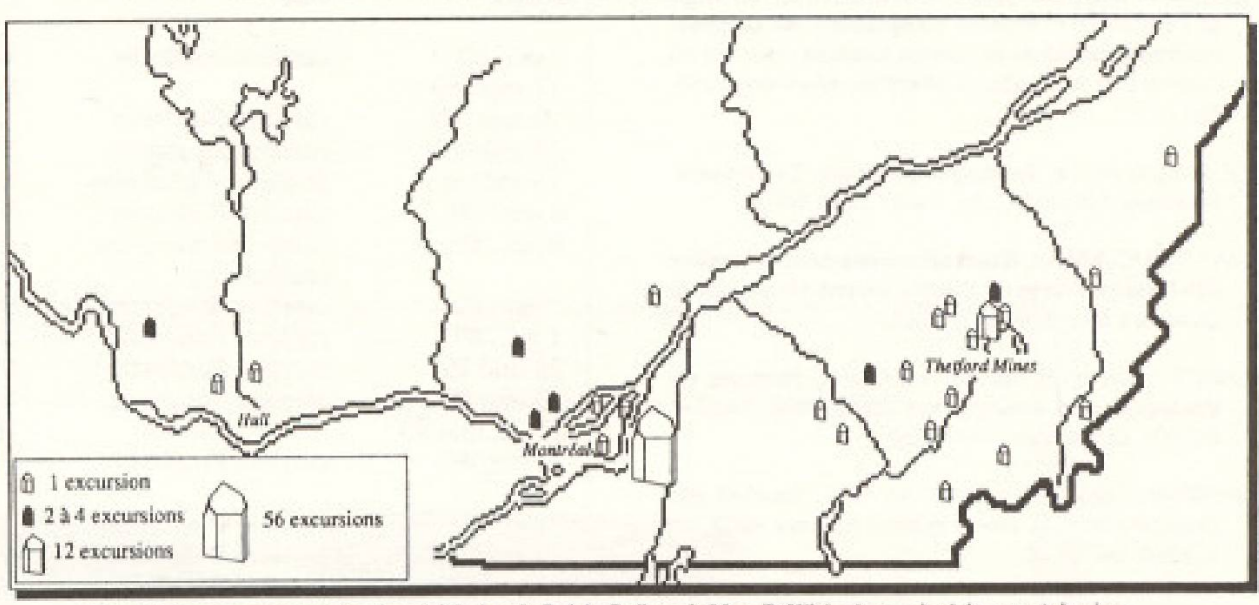

Comme le montre cette carte des siles visiles dans le Sud du Quebec, le Mons St-Hilaire (gros cristal de quanz) domine nettement parni les destinations de collecte awoe plas de 56 eveursions depuis 1986.

TABLEAU 1

Évolution du membership au Montreal Gem \& Mineral Club

\begin{tabular}{|c|c|c|c|c|c|c|c|}
\hline Année & $A$ vie & $\begin{array}{c}\text { Honori- } \\
\text { fique } \\
\text { (annuel) }\end{array}$ & $\begin{array}{l}\text { Institu- } \\
\text { tionnel }\end{array}$ & Famille & Adulte & Jeune & Total \\
\hline 1989 & 6 & 6 & 35 & 46 & 121 & 6 & 220 \\
\hline 1988 & 6 & 6 & 35 & 54 & 146 & 8 & 255 \\
\hline 1987 & 6 & 8 & 36 & 63 & 133 & 2 & 248 \\
\hline 1986 & & & & 33 & 71 & & 104 \\
\hline 1985 & & & & 28 & 60 & 3 & 91 \\
\hline 1984 & & & & 25 & 49 & & 74 \\
\hline \multicolumn{8}{|l|}{ de 1976} \\
\hline à 1983 & & & & & & & p.d. \\
\hline 1975 & 3 & & & & 85 & & 88 \\
\hline 1974 & & & & & & & p.d. \\
\hline 1973 & & & & & & & p.d. \\
\hline 1972 & & & & & 145 & & 145 \\
\hline 1971 & 5 & 1 & & & 171 & & 177 \\
\hline 1970 & & & & & & & p.d. \\
\hline 1969 & & & & & & & p.d. \\
\hline 1968 & & & & & & & p.d. \\
\hline 1967 & 1 & & & & 79 & & 80 \\
\hline 1966 & & 3 & & & 90 & & 93 \\
\hline 1965 & & & & 16 & 38 & 19 & 73 \\
\hline 1964 & & & & 20 & 42 & 27 & 89 \\
\hline 1963 & & & & & 79 & & 79 \\
\hline 1962 & & & & & & & p.d. \\
\hline 1961 & & & & & & & p.d. \\
\hline 1960 & & & & & 61 & & 61 \\
\hline
\end{tabular}


BOIVIN, D.J., La restauration et le réaménagement des mines et carrières abandonnées au Québec méridional, thè se de maltrise deposéte au programme d'Amenagement du territcire et de developpement regional, Untwersite Laval, 1981, 230 p. et annexes.

BRILLANT, Jean, LAPOINTE, Andre, OCCHIETTI, Serge et Aésl PATAY, Pierres sculptables du Québec, marbres, calcaires et roches tendres. Presses de I'Université du Québec à Montrésal, Montréal, 1986, $181 \mathrm{p}$.

CAILLEUX, André, Géologie gènérale, Terre-LunePlanetes, Masson-Fides, Paris, 1976, $346 \mathrm{p}$.

DIVERGILIO, Michel, Illustrations des genres fossiles des Basses-Terres du Saint-Laurent, Universite du Québec a Montréal, 1978, $91 \mathrm{p}$.

LANDRY, Brung et Michel MERCIER, Notions de géologie avec exemplaires du Québec, Modulo Editeur, Outremont, 1983, $426 \mathrm{p}$.

MURPHY, Peter E. et Robin BAYLEY, Towism and Disaster Flanning Geographieal Review, vol. 7, no 1. $1989, p p .36-46$

PELLEAIN, Jean-Guy et Therrese SEGUIN, Répertoine des principaux sites fossiliferes du Québec pour le palétontologue amateur, 2 ème édition, Fos.giles Plug. Enr, Montréal, 1988, 154 p.

ROBINSON, Éric, Geological Conservation, Mine \& Quarry Environment wol. 3, no 1, 1989, pp. 28-30.

SABINA, Ann P., Roeks and Minerals for the Collector: Eastern Townships and Gaspé, Quebec and Parts of New-Brunswick, Paper 66-51, Dept. of Energy, Mines \& Resources, 1975, 2nd Edition, Ottawa, $1975 \mathrm{a}, 170 \mathrm{p}$.

SABINA, Ann P., Rocks and Minerals for the Collector: Kingston, Ontario to Lac St-Jean, Québec, Paper 67-51, Dept. of Energy, Mines \& Rescurces, 2nd Edition, Ottawa, 1975b. $147 \mathrm{p}$.

\section{ANNEXE}

Cette annexe donne le nom et l'adresse des clubs de géologues amateurs au Québec ainsi que le nombre actuel de leurs membres.

Club de Minéralogie de Montréal

C.P. 305, suoc. St-Michel

Montréal (Québec)

H2A $3 \mathrm{M} 1$

fondé en 1977

actuellement 307 membres

Montreal Gem \& Mineral Club

P.O. Box 1717, Station B

Montréal (Québec)

H3B 3L3

fondé en 1957

actuellement 220 membres

Société Minéralogique de la région de l'amiante

a/s Monsieur Pierre Laroche

C.P. 462

Thetford Mines (Québec)

G6G 5T3

fondé en 1974

maintenant associé au groupe Les amis du musbé actuellement 250 membres
TABLEAU 2

Excursions minéralogiques du Club de Minéralogié de Montréal

\begin{tabular}{|c|c|c|c|c|}
\hline Dates & Site & Lieu & Région Par & ants \\
\hline 8 oct. 89 & carrière Poudrette & St-Hilaire & Montérégie & 60 \\
\hline 17 sept. 89 & & Pierrepont & New York & 10 \\
\hline 10 sept. 89 & carrière Poudrette & St-Hilaire & Montérégie & 82 \\
\hline 27 août 89 & carrière Poudrette & St-Hilaire & Montérégie & 64 \\
\hline 13 août 89 & St-Rémi China clay & St-Rémi-d'Amherst & Laurentides & 18 \\
\hline 6 août 89 & carrière Poudrette & St-Hilaire & Montérégie & 63 \\
\hline 9 juil. 89 & $\begin{array}{l}\text { Mine St-Lawrence } \\
\text { columbium }\end{array}$ & Oka & Montréal & 18 \\
\hline 2 juil. 89 & carrière Poudrette & St-Hilaire & Montérégie & 47 \\
\hline 1 juil. 89 & carrière Poudrette & St-Hilaire & Montérégie & 88 \\
\hline $28 \mathrm{mai} 89$ & carrière Poudrette & St-Hilaire & Montérégie & 43 \\
\hline 27 mai 89 & carriere Poudrette & Si-Hilaire & Montérégie & 79 \\
\hline $20-22$ mai 89 & & Herkimer & New York & 12 \\
\hline 14 mai 89 & carrière Poudrette & St-Hilaire & Montérégie & 82 \\
\hline 9 oct. 88 & carrière Poudrette & St-Hilaire & Montérégie & 58 \\
\hline 18 sept. 88 & carrière Poudrette & St-Hilaire & Montérégie & 42 \\
\hline 21 août 88 & carrière Poudrette & St-Hilaire & Montérégie & 42 \\
\hline 24 juil. 88 & carrière Poudrette & St-Hilaire & Montéregie & 35 \\
\hline 16 juil. 88 & Mine Jeffrey & Asbestos & Estrie & 23 \\
\hline 3 juil. 88 & carrière Poudrette & St-Hilaire & Montérégiè & 38 \\
\hline 2 juil. 88 & carrière Poudrette & St-Hilaire & Montérégie & 62 \\
\hline 26 juin 88 & & Black Lake & Estrie & 26 \\
\hline 19 juin 88 & carrière Francon & Montréal & Montréal & 30 \\
\hline 12 juin 88 & carrière Poudrette & St-Hilaire & Montérégie & 43 \\
\hline 29 mai 88 & carrière Poudrette & St-Hilaire & Montérégie & 57 \\
\hline 28 mai 88 & carrière Poudrette & St-Hilaire & Montérégie & 99 \\
\hline 1 mai 88 & carriere Poudrette & St-Hilaire & Montérégie & 88 \\
\hline $10-12$ oct. 87 & & Herkimer & New York & \\
\hline 13 sept. 87 & carrière Poudrette & St-Hilaire & Montérégie & 66 \\
\hline 30 août 87 & carrière Montréal-Est & Montréal-Est & Montréal & 22 \\
\hline 9 août 87 & carrière Poudrette & St-Hilaire & Montérégie & 54 \\
\hline $1-2$ août 87 & & Bancroft & Ontario & p.d. \\
\hline $30-31$ juil. 87 & & Bancroft & Ontario & p.d. \\
\hline 26 juil. 87 & Orford Nickel mine & St-Denis-de-Brompton & Estrie & p.d. \\
\hline $25-26$ juil. 87 & & Wilberforce & Ontario & p.d. \\
\hline $11-12$ juil. 87 & & Pierrepont & New York & p.d. \\
\hline 4 juil. 87 & carrière Poudrette & St-Hilaire & Montérégie & 72 \\
\hline 5 juil. 87 & carrière Poudrette & St-Hilaire & Montérégie & 52 \\
\hline 28 juin 87 & & Black Lake & Estrie & 20 \\
\hline 14 juin 87 & carrière Poudrette & St-Hilaire & Montérégie & 58 \\
\hline 23 mai 87 & carrière Poudrette & St-Hilaire & Montérégie & 65 \\
\hline 24 mai 87 & carrière Poudrette & St-Hilaire & Montérégie & 47 \\
\hline $16-18$ mai 87 & & Herkimer & New York & 12 \\
\hline 26 oct. 86 & carrière Poudrette & St-Hilaire & Montéré & 30 \\
\hline 5 oct. 86 & carrière Poudrette & St-Hilaire & Montérégie & 42 \\
\hline 14 sept. 86 & & St-Pierre deWakefield & Outaouais & 7 \\
\hline 7 sept. 86 & carrière Poudrette & St-Hilaire & Montérégie & 40 \\
\hline $30-31$ sept. 86 & Mine Yates & Otter Lake & Outaouais & 6 \\
\hline 17 août 86 & Mine St-Lawrence & $\begin{array}{l}\text { Oka } \\
\text { columbium }\end{array}$ & Montréal & 5 \\
\hline & & Bancroft & Ontario & 6 \\
\hline 10 août 86 & carrière Poudrette & St-Hilaire & Montérégie & 30 \\
\hline 20 juil. 86 & Orford Nickel mine & St-Denis-de-Brompton & Estrie & 6 \\
\hline 13 juil. 86 & carrière Poudrette & St-Hilaire & Montérégie & 39 \\
\hline 6 juil. 86 & & Black Lake & Estrie & 20 \\
\hline 22 juin 86 & carrière Poudrette & St-Hilaire & Montérégie & 37 \\
\hline 15 juin 86 & & Pierrepont & New York & 11 \\
\hline 8 juin 86 & carrière Mathers & St-Eustache & Montréal & 41 \\
\hline 1 juin 86 & carrière Mathers & St-Eustache & Montréal & 6 \\
\hline $4 \mathrm{mai} 86$ & carrière Poudrette & St-Hilaire & Montérégie & 32 \\
\hline 1985 & Mine Jeffrey & Asbestos & Estrie & 25 \\
\hline
\end{tabular}

p.d.: pas de données 
TABLEAU NO 3

Excursions minéralogiques du Montreal Gem \& Mineral Club

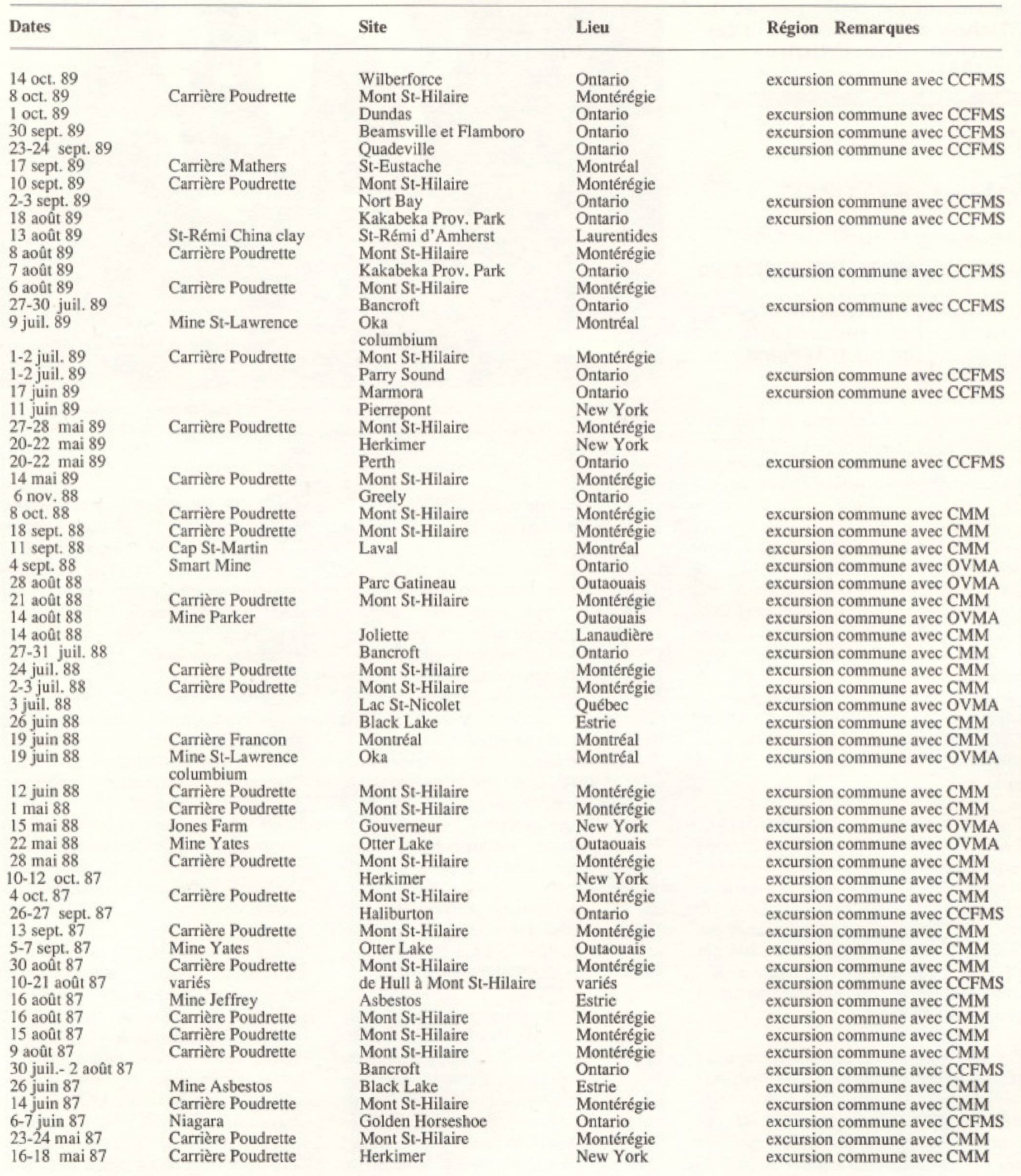

CMM: Club de Minéralogie de Montréal; CCFMS: Central Canadian Federation of Mineralogical Societies; OVMA: Ottawa Valley Mineral Association. 